\section{Análisis de falsos negativos en la cintigrafía SPECT de paratiroides con sestamibi en pacientes con hiperparatiroidismo primario sometidos a cirugía entre 2008-2015 en hospital universitario}

\author{
GABRIELA PAILLAHUEQUE ${ }^{1, \mathrm{a}}$, TERESA MASSARDO ${ }^{1}$, \\ MARCELA BARBERÁN ${ }^{2}$, GERSON OCARES ${ }^{2, b}$, IVÁN GALLEGOS ${ }^{3}$, \\ LUIS TORO $^{4,5}$, A. VERÓNICA ARAYA ${ }^{2}$
}

\section{False negative spect parathyroid scintigraphy with sestamibi in patients with primary hyperparathyroidism}

Background: ${ }^{99 m}$ Tc-sestamibi parathyroid SPECT scintigraphy is a useful tool in the pre-operative study of hyperparathyroidism. False negatives (FN) have been reported in 5.7-14\% of the examinations. Aim: To characterize ${ }^{99 m} \mathrm{Tc}$-sestamibi FN in cases referred for primary hyperparathyroidism $(\mathrm{PHP})$ to a university hospital. Material and Methods: Descriptive retrospective analysis. We included patients with PHP, studied with SPECT scintigraphy, operated at our center between 2008 and 2015. Clinical and surgical data were recorded; biopsies of the FN were blindly reviewed by one pathologist.Results: One hundred twenty one scintigraphies fulfilled the inclusion criteria. Seven (5.8\%) were negative and 114 positive. There was no difference in age, sex and PTH levels between FN and true positive scintigraphies. At surgery, one FN case had two hyperplasic glands and two cases had ectopic glands. Pathology reported adenoma in three cases, hyperplasia in three and carcinoma in one. The largest diameter of the lesion was lower in FN (1.3 and $2.1 \mathrm{~cm}$ respectively, $p=0.02$ ) and the proportion of adenomas was higher in true positive cases (29\% and $75 \%$ respectively; $p<0.01)$. The interval between scintigraphy and parathyroidectomy was greater in FN with a median of 92 days (range 20 days-3.2 years, $p<0.01)$. The percentage of oxyphilic cells observed was similar in both groups. Conclusions: FN parathyroid SPECT scintigraphies in PHP are uncommon. They corresponded to lesions under the equipment's resolution limit and resulted in longer time lags between scintigraphy and surgery.

(Rev Med Chile 2017; 145: 1021-1027)

Key words: Hyperparathyroidism, Primary; Parathyroid Neoplasms; Parathyroidectomy; Technetium Tc 99m Sestamibi.

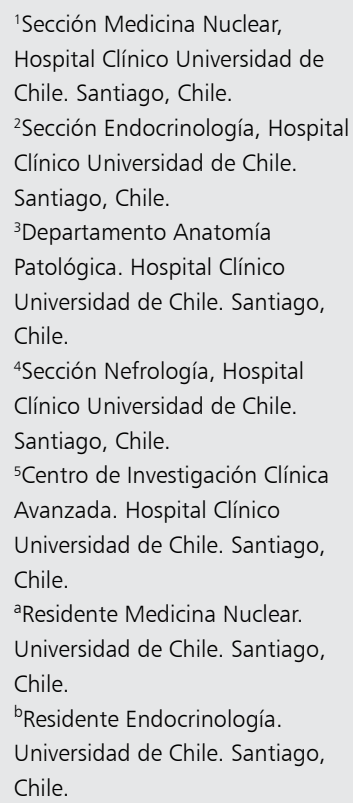

Recibido el 20 de marzo de 2017 aceptado el 9 de agosto de 2017

Correspondencia a:

Verónica Araya Quintanilla. Sección Endocrinología Hospital Clínico Universidad de Chile.

Santos Dumont 999.

Independencia. Santiago, Chile. varaya@hcuch.cl
$\mathrm{E}$ 1 hiperparatiroidismo primario (HPP) se caracteriza por la presencia de hipercalcemia asociada a niveles elevados o inapropiadamente normales de hormona paratiroidea (PTH).
Actualmente, la mayoría de los pacientes son asintomáticos al momento del diagnóstico, pudiendo presentar grados variables de compromiso óseo, renal, neuropsicológico, cardiovascular y gastroin- 
testinal ${ }^{1,2}$. Tiene una incidencia de 16 a 95 casos cada 100.000 habitantes por año dependiendo de la población estudiada y afecta principalmente a mujeres entre 40 y 60 años, con relación mujer/ hombre 2-5:13. La causa más frecuente es adenoma único $(80 \%)$, menos frecuente hiperplasia de las 4 glándulas (2 a 5\%) y carcinoma de paratiroides $(0,7 \%)^{1,4}$.

En los casos con indicación quirúrgica, es imprescindible localizar correctamente la glándula afectada, con el fin de realizar cirugía mínimamente invasiva, disminuyendo el tiempo operatorio y complicaciones, con alta hospitalaria temprana ${ }^{5,6}$. El cintigrama SPECT (tomografía de emisión de fotón único) de paratiroides Tc99m-sestamibi es un método útil para la detección y localización preoperatoria de glándulas anormales, inclusive ectópicas ${ }^{5}$.

El sestamibi es un compuesto isonitrilo catiónico monovalente lipofílico que difunde pasivamente a través de la membrana celular, siendo secuestrado por las mitocondrias ${ }^{7}$, que son especialmente abundantes en las glándulas paratiroideas hiperfuncionantes. Esto provoca un aumento de captación del radiotrazador en la imagen temprana, que persiste en adquisición tardía; a diferencia de la glándula tiroides, la cual tiene lavado precoz. La técnica presenta mejor sensibilidad para adenoma único de localización habitual $(88 \%)$ y de mayor peso ${ }^{4}$. La sensibilidad disminuye en tumores pequeños y enfermedad multiglandular pero, se puede aumentar con el método SPECT-CT (SPECT-tomografía computada $)^{8}$. La expresión de glicoproteína-P (P-gp) y la presencia de células oxifílas también podrían afectar el grado de captación. La P-gp es una proteína de transmembrana codificada por el gen MDR. Las glándulas con mayor expresión no acumulan radiotrazador, produciendo falsos negativos ${ }^{9,10}$. Las células oxífilas, son ricas en mitocondrias por lo tanto, los tumores con mayor porcentaje de estas células tendrían una adecuada captación del sestamibi ${ }^{11}$.

En la literatura, se han descrito falsos negativos (FN) en 5,7-14\% ${ }^{12,13}$ de los cintigramas paratiroideos realizados en pacientes con HPP con indicación quirúrgica. Nuestro estudio tiene como objetivo caracterizar los casos de HPP referidos a nuestro centro, que resultaron FN en el cintigrama SPECT de paratiroides con Tc99m-sestamibi.

\section{Material y Método}

Análisis descriptivo retrospectivo de pacientes con HPP diagnosticado. Criterios de inclusión: hipercalcemia y hormona paratiroidea intacta (iPTH) elevada o inapropiadamente normal, estudiados con cintigrama paratiroideo y operados entre 2008-2015 en nuestro centro. Se excluyeron: casos con hiperparatirodismo secundario o terciario, y aquellos con información incompleta en la ficha.

En el cintigrama paratiroideo se utilizó sestamibi marcado con Tecnecio-99m, usando técnica doble fase, con adquisición de imágenes planares y tomográficas, con doble trazador en gamma cámara Siemens Ecam.

La adquisición se realizó en proyección anteroposterior con extensión cervical, comprendiendo el área que va desde las glándulas salivales hasta el mediastino (incluyendo corazón). A los $10 \mathrm{~min}$ post inyección del Tc99m-sestamibi $555-740 \mathrm{MBq}$ (15-20 mCi), se efectuó imagen estática de $10 \mathrm{~min}$ de duración, inmediatamente después se realizó imagen SPECT utilizando gamma cámara con sistema de doble detector, con colimadores paralelos de baja energía y alta resolución. A las $2 \mathrm{~h}$ se realizó nueva estática de $10 \mathrm{~min}$, posteriormente se inyectaron $555 \mathrm{MBq}(15 \mathrm{mCi})$ de Tc99m-pertecneciato sin mover el paciente, para luego de $15 \mathrm{~min}$, adquirir otra estática de $10 \mathrm{~min}$. Las imágenes planares se dispusieron en forma comparativa, incluyendo estática inicial con sestamibi, lavado de 2 h y tardía de pertecneciato. Reconstrucción de imágenes SPECT en eje coronal, sagital y transversal con método iterativo.

Se interpretó como estudio positivo para tejido paratiroideo hiperfuncionante la presencia de hipercaptación focal del radiotrazador en la imagen temprana, que persiste en imágenes tardías $(2 \mathrm{~h})$; esto asociado a la localización tridimensional en imágenes SPECT. Se informó como estudio negativo, a la ausencia de focos de hipercaptación del radiotrazador que se mantuvieran en imágenes tardías (Figura 1).

Se analizaron datos clínicos y protocolos operatorios de los verdaderos positivos (VP) y falsos negativos (FN). Las biopsias de los FN fueron revisadas por un mismo patólogo en forma ciega, evaluando presencia y porcentaje de células oxífilas con tinción de hematoxilina-eosina. Se estableció como punto de corte para el análisis, presencia en 


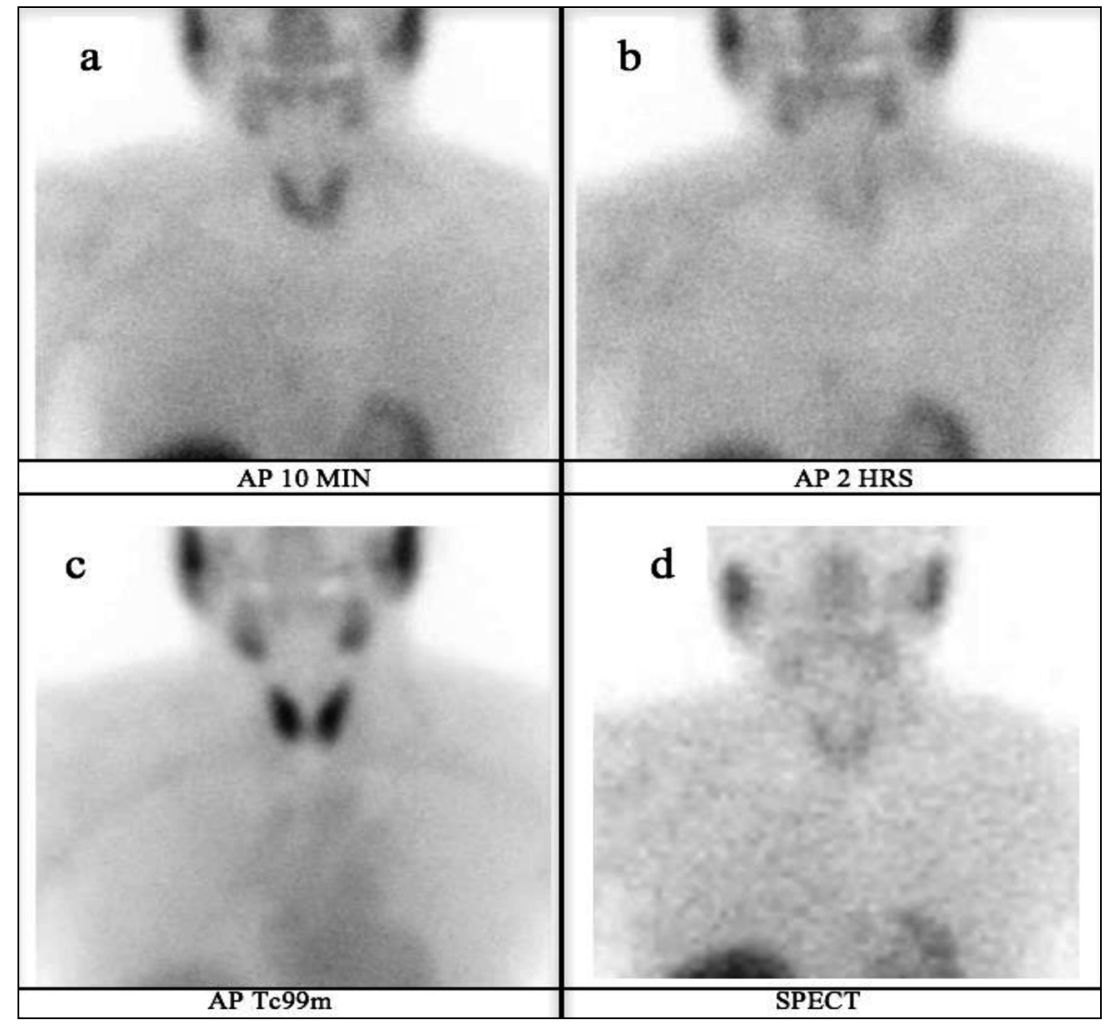

Figura 1. Cintigrama paratiroideoTc99m-sestamibi. Se muestran imágenes planares AP comparativas que incluyen estática inicial a los 10 min post inyección del Tc99m-sestamibi (a), control a las $2 \mathrm{~h}$ (b) tardía post inyección Tc99m-Pertecneciato (c) y SPECT en 3 dimensiones desde AP (d). La adquisición comprende el área desde las glándulas salivales hasta el mediastino (incluyendo corazón). Paciente femenino de 53 años, con estudio cintigráfico negativo para tejido paratiroideo hiperfuncionante, en la cual se confirmó la presencia de adenoma paratiroideo retroesofágico derecho en la cirugía y biopsia posterior.
$30 \%$ de la muestra. Este estudio fue aprobado por el Comité Ético Científico del Hospital Clínico de la Universidad de Chile.

Análisis estadístico: Las variables continuas se expresan como media aritmética \pm desviación estándar (si distribución paramétrica) o mediana [percentil 25 - percentil 75] (si distribución no paramétrica). Se evaluó distribución mediante prueba de Shapiro-Wilk. Para el contraste de hipótesis se utilizó prueba exacta de Fisher para variables categóricas, prueba $\mathrm{t}$ de Student de muestras no pareadas o prueba U de Mann-Whitney, para variables continuas. Todos los análisis fueron de 2 colas. Se consideró como diferencia estadísticamente significativa un valor $\mathrm{p}$ menor al 5\% $(\mathrm{p}<0,05)$.

\section{Resultados}

De 382 cintigramas paratiroideos solicitados por diversas causas en el período, 121 cumplieron criterios para ingresar al estudio. De ellos, 7 casos fueron negativos (5,8\%). Las características clínicas, de laboratorio e histológicas de los VP y FN se muestran en la Tabla 1 . No hubo diferencia entre ambos grupos para edad, sexo y valor de iPTH pero, la calcemia fue significativamente mayor en $\operatorname{los} \mathrm{FN}(12,7 \pm 2,7$ vs $11,5 \pm 0,5 \mathrm{mg} / \mathrm{dL} ; \mathrm{p}<0,001)$. En la cirugía se encontró en uno de los FN 2 glándulas hiperplásicas y en otros dos, glándulas ectópicas. La histología demostró: adenoma (3), hiperplasia (3), carcinoma paratiroideo (1). La proporción de adenomas fue significativamente menor en los FN (29\% vs $75 \%$; p = 0,007). El diámetro mayor de la lesión fue significativamente menor en los FN ( 1,3 vs $2,1 \mathrm{~cm}, \mathrm{p}=0,02)$ (Figura 2). El lapso entre realización del cintigrama y la paratiroidectomía fue significativamente mayor en los FN (mediana: 92 días [rango: 20 días-3.2 años], $\mathrm{p}<0,001$ )(Figura 3). No hubo diferencias en el porcentaje de células oxífilas. Además, cuatro casos presentaban bocio multinodular y uno tiroiditis de Hashimoto. Ver datos individuales en Tabla 2. 
Tabla 1. Características clínicas, de laboratorio e histología de pacientes con hiperparatiroidismo primario y cintigrama SPECT de Paratiroides con sestamibi

\begin{tabular}{|c|c|c|c|}
\hline & cintigrama (-) FN & cintigrama (+) VP & Valor $\mathbf{p}^{*}$ \\
\hline Número pacientes, n (\%) & $7(6 \%)$ & $114(94 \%)$ & \\
\hline Edad (años) & $54,4 \pm 6,0$ & $52,7 \pm 13,3$ & 0,76 \\
\hline Sexo Femenino, n (\%) & $6(86 \%)$ & $100(87 \%)$ & 0,87 \\
\hline Calcemia (mg/dL) & $12,7 \pm 2,7$ & $11,5 \pm 0,5$ & $<0,001$ \\
\hline iPTH (pg/mL) & $128[120-315]$ & $154,8[95,5-283,3]$ & 0,51 \\
\hline Lapso Cintigrama/Paratiroidectomía (días) & $92[63-502]$ & $59[39-110]$ & $<0,001$ \\
\hline Tamaño máximo (cm) & $1,3[1,0-1,5]$ & $2,1[1,4-2,6]$ & 0,02 \\
\hline \multicolumn{4}{|l|}{ Tipo histológico, n (\%) } \\
\hline Adenoma & $2(29 \%)$ & $86(75 \%)$ & 0,007 \\
\hline No Adenoma** & $5(71 \%)$ & $28(25 \%)$ & \\
\hline \multicolumn{4}{|l|}{ \% Células oxifílicas } \\
\hline$<30 \%$ & $5(71 \%)$ & $72(63 \%)$ & 0,65 \\
\hline$\geq 30 \%$ & $2(29 \%)$ & $42(37 \%)$ & \\
\hline
\end{tabular}

Las variables se expresan como media aritmética \pm desviación estándar, o mediana [percentil 25 - percentil 75]. *Cintigrama (-) versus cintigrama $(+) .{ }^{* *}$ Incluye: hiperplasias, ectópicos, carcinomas.

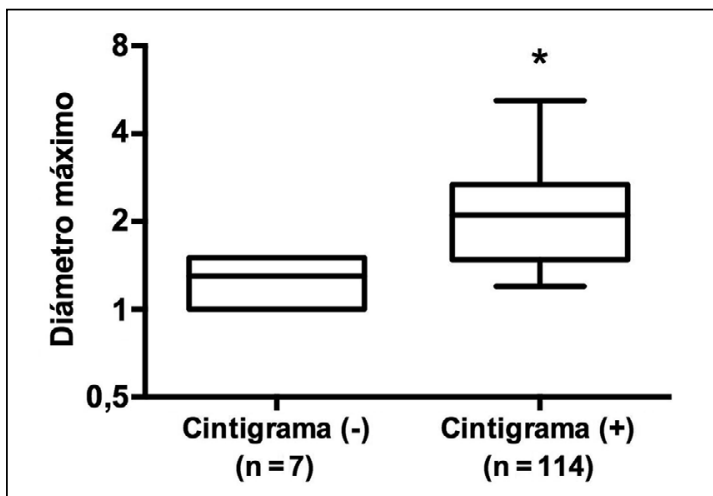

Figura 2. Diámetro máximo $(\mathrm{cm})$ de la lesión paratiroidea extirpada. Se observó diferencia significativa entre cintigramas positivos $(+)$ o verdaderos positivos y cintigramas negativos (-) o falsos negativos, ${ }^{*} p=0,02$.

\section{Discusión}

El cintigrama SPECT paratiroideo Tc99m-sestamibi ofrece alta sensibilidad y escasos falsos negativos, menor al 15\% en pacientes con HPP, por lo cual es utilizado para localizar la glándula patológica, realizar cirugías mínimamente invasivas y evitar fracasos.

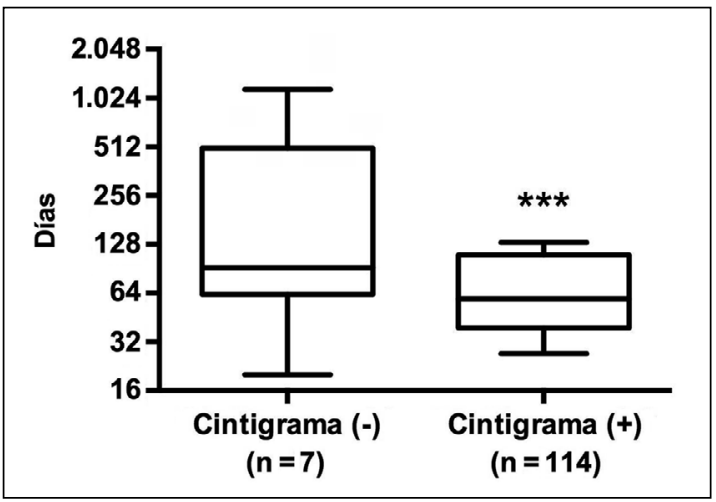

Figura 3. Lapso (días) entre la realización del cintigramay la paratiroidectomía. Se observó diferencia significativa entre cintigramas positivos $(+)$ o verdaderos positivos y cintigramas negativos (-) o falsos negativos, ${ }^{* * *} p<0,001$. Nota: escala logarítmica en eje $Y$ (días).

En nuestra experiencia, el porcentaje de FN del cintigrama paratiroideo en pacientes con HPP confirmado bioquímicamente, fue similar a lo descrito en otras publicaciones ${ }^{12,13}$. El factor más comúnmente reportado como causa de falsos negativos es el tamaño de la glándula paratiroidea. Las glándulas más pequeñas tienen menos probabilidades de ser detectadas ${ }^{7,8,14}$, lo cual es con- 
Tabla 2. Pacientes con hiperparatiroidismo primario y falso negativo en el cintigrama SPECT de Paratiroides con sestamibi

\begin{tabular}{|ccccccccc|}
\hline $\begin{array}{c}\text { Edad } \\
\text { (años) }\end{array}$ & Sexo & $\begin{array}{c}\text { Calcemia }^{\dagger} \\
(\mathbf{m g} / \mathbf{d l})\end{array}$ & $\begin{array}{c}\text { PTH } \\
(\mathbf{p g} / \mathbf{m L})\end{array}$ & $\begin{array}{c}\text { Lapso C/P } \\
\text { (días) }\end{array}$ & $\begin{array}{c}\text { Tipo } \\
\text { histológico }\end{array}$ & $\begin{array}{c}\text { Ubicación } \\
\text { Tamaño }\end{array} \begin{array}{c}\text { Células } \\
\text { oxífilas }\end{array}$ \\
\hline 54 & F & 11,2 & 93,3 & 236 & Adenoma & Retroesofágico & $15 \times 8$ & $<10 \%$ \\
\hline 46 & F & 12,2 & 256 & 72 & Hiperplasia & Retroesofágico & 15 & $30 \%$ \\
\hline 57 & F & 11,5 & 125 & 1156 & Adenoma & II & 10 & $<10$ \\
\hline 63 & F & 12 & 315 & 92 & Carcinoma & II & $13 \times 10$ & $60 \%$ \\
\hline 56 & M & 11,1 & 120 & 502 & Adenoma & SD & $15 \times 10 \times 6$ & $<10 \%$ \\
\hline 58 & F & 12,3 & 365 & 63 & Hiperplasia & SD & $11 \times 7$ & $0 \%$ \\
\hline
\end{tabular}

F: femenino, M: masculino, ${ }^{\dagger}$ Calcemia preoperatoria. C/P: cintigrama-paratiroidectomía. II: inferior izquierdo, SD: superior derecho, ID: inferior derecho, SI: superior izquierdo.

cordante con nuestro estudio, en que las lesiones fueron significativamente más pequeñas que los VP (1,3 vs $2,1 \mathrm{~cm})$, bajo el límite de resolución del equipo. Además, el lapso de tiempo entre la realización de la imagen isotópica y cirugía fue más prolongado que en los pacientes con estudios positivos, por razones no médicas. Esto pudo determinar la discrepancia entre el resultado negativo del cintigrama y el tamaño confirmado por biopsia, ya que no fue posible contar con un nuevo estudio antes de la cirugía.

Hay que señalar que la gammagrafía es menos sensible para enfermedad paratiroidea multiglandular e hiperplasia. En este estudio, de los tres casos FN con hiperplasia, uno tenía dos glándulas hiperplásicas y además enfermedad tiroidea multinodular otro, un tamaño glandular pequeño y en el tercero, la glándula era de situación ectópica. Por este motivo, en pacientes con diagnóstico bioquímico de HPP e imágenes cintigráficas que no muestran un área con captación sospechosa, debe considerarse la posibilidad de hiperplasia, la que puede ser multiglandular ${ }^{7,15}$.

Recientemente un grupo evaluó la localización anatómica de las glándulas paratiroides en los exámenes de imágenes, previo a la cirugía, proponiendo el uso de la clasificación de Perrier para informar el cintigrama sestamibi SPECT-CT ${ }^{16}$. En este estudio se demostró que la localización descrita en las imágenes SPECT-CT preoperatorias presenta un $80 \%$ de concordancia con el resultado postquirúrgico, sugiriendo que el uso de esta clasi- ficación en la descripción de las imágenes aumenta la probabilidad de hallazgo del tejido anormal ${ }^{14}$.

Por otra parte, el nivel de iPTH también se ha correlacionado con la visualización de las paratiroides en la gammagrafía sestamibi, logrando mejor identificación cuando los niveles están sobre $200 \mathrm{pg} / \mathrm{ml}^{17}$. Aunque no hubo diferencia significativa entre los VP y FN, en 4 de estos pacientes, la iPTH fue $<200 \mathrm{pg} / \mathrm{ml}$, lo cual podría haber contribuido a la ausencia de visualización de las glándulas. En cambio, la calcemia fue significativamente mayor en los FN lo cual indica que no habría relación del nivel de calcio con el tamaño o tipo de lesión.

Si bien la evidencia aún es controversial, se ha demostrado que la presencia de células oxífilas en mayor concentración, determinaría una adecuada captación del sestamibi. Sin embargo, en nuestro estudio, no observamos diferencia entre los VP y FN en cuanto al porcentaje de células oxífilas ${ }^{11}$. Por el contrario, el carcinoma presentó el porcentaje más alto lo que indicaría que hay otros factores involucrados. Entre estos, la mayor expresión de P-gp en tumores malignos, impediría la acumulación del radiotrazador, determinando falsos negativos ${ }^{9,10,18}$.

A pesar de su alta sensibilidad y especificidad, el SPECT está limitado por una baja resolución espacial e incapacidad para proporcionar detalles anatómicos. Los sistemas de modalidad dual (SPECT-CT) permiten realizar imágenes tanto funcionales como estructurales, que mejoran la 
calidad y entregan más detalles sobre ubicación, tamaño y morfología de la lesión, siendo la imagen SPECT-CT superior a SPECT, especialmente en glándulas $<10 \mathrm{~mm}$ y adenomas $<200 \mathrm{mg}^{6,19-21}$. Sin embargo, el SPECT-CT requiere disponibilidad del equipo, tiempo de imagen adicional y mayor radiación ${ }^{8}$.

Por otra parte, se ha planteado el uso de SPECT con colimador pinhole para aumenta la resolución espacial y sensibilidad del cintigrama, sin embargo, prolonga el tiempo del estudio y no reemplaza las imágenes planares, por su menor campo de visión ${ }^{22}$.

Se ha demostrado que la presencia de imágenes negativas influye en la conducta terapéutica. En un estudio de un grupo quirúrgico, publicado recientemente, se observó que los endocrinólogos tenían menos probabilidades de derivar al cirujano a los pacientes con cintigrama paratiroideo negativo ( $\mathrm{OR}=0,36$; IC 95\%: 0,18-0,73), así como los cirujanos estaban menos inclinados a realizar paratiroidectomía en estos pacientes $(\mathrm{OR}=0,32 \text {; IC 95\%: 0,11-0,91 })^{12}$. En estos casos, el cintigrama paratiroideo y la ecotomografía pueden ser complementarios, sobre todo para la localización de glándulas ectópicas retroesofágicas o intratiroideas ${ }^{23,24}$. También se puede considerar el uso de FDG (fluordeoxiglucosa) con técnica PET-CT (tomografía de emisión de positrones) como modalidad de imágenes de segunda línea para localización de la glándula patológica, pues disminuye los falsos negativos ${ }^{25}$. Así mismo, se han descrito otros radiotrazadores PET, como C11-metionina y F18-fluorcolina, esta última disponible en nuestro país ${ }^{26,27}$.

Una de las debilidades de este trabajo es corresponder a una revisión retrospectiva y que no se contó con la información clínica de todos los cintigramas realizados debido a que, la población que se atiende en nuestro centro no es cautiva. Como fortaleza, podemos mencionar que corresponde a la práctica clínica real y que obtuvimos un porcentaje de FN similar a lo publicado internacionalmente.

En conclusión, los falsos negativos del SPECT paratiroideo en pacientes con HPP fueron escasos, similar a lo descrito en la literatura, correspondiendo a lesiones pequeñas, bajo el límite de resolución del equipo. Además, el hallazgo de lesiones ectópicas, hiperplasia y cáncer paratiroideo, también pueden explicar la dificultad en su pesquisa, apuntando a que existen otros factores, como la escasa presencia de células oxífilas o una mayor expresión de la P-gp que contribuyen al resultado negativo. El uso de nuevas técnicas con imágenes híbridas, puede mejorar la localización anatómica de las glándulas comprometidas pero, finalmente es el diagnóstico bioquímico y el criterio clínico lo que determina la conducta terapéutica.

\section{Referencias}

1. Silverberg SJ, Bilezikian JP. The diagnosis and management of asymptomatic primary hyperparathyroidism. Nat Clin Pract Endocrinol Metab 2006; 2: 494-503.

2. Silverberg SJ, Clarke BL, Peacock M, Bandeira F, Boutroy S, Cusano NE, et al. Current issues in the presentation of asymptomatic primary hyperparathyroidism: proceedings of the Fourth International Workshop. Clin Endocrinol Metab 2014; 99: 3580-94.

3. Minisola S, Pepe J, Scillitani A, Cipriani C. Explaining geographical variation in the presentation of primary hyperparathyroidism. Lancet Diabetes Endocrinol 2016; 4: 641-3.

4. Ruda JM, Hollenbeak CS, Stack BC Jr. A systematic review of the diagnosis and treatment of primary hyperparathyroidism from 1995 to 2003.Otolaryngol Head Neck Surg 2005; 132: 359-72.

5. Udelsman R, Åkerström G, Biagini C, Duh QY, Miccoli $\mathrm{P}$, Niederle B, et al. The surgical management of asymptomatic primary hyperparathyroidism: proceedings of the Fourth International Workshop. J Clin Endocrinol Metab 2014; 99: 3595-606.

6. Sandqvist P, Nilsson IL, Grybäck P, Sánchez-Crespo A, Sundin A. SPECT/CT's Advantage for Preoperative Localization of Small Parathyroid Adenomas in Primary Hyperparathyroidism. Clin Nucl Med 2017; 42: 109-14.

7. Eslamy HK, Ziessman HA. Parathyroid Scintigraphy in Patients with Primary Hyperparathyroidism: 99m-Tc Sestamibi SPECT and SPECT/CT. Radio Graphics 2008; 28: 1461-76.

8. Vaz A, Griffiths M. Parathyroid imaging and localization using SPECT/CT: initial results. J Nucl Med Technol 2011; 39: 195-200.

9. Sun SS, Shiau YC, Lin CC, Kao A, Lee CC. Correlation between $\mathrm{P}$-glycoprotein ( $\mathrm{P}$-gp) expression in parathyroid and Tc-99m MIBI parathyroid image findings. Nucl Med Biol 2001; 28: 929-33.

10. Gupta Y, Ahmed R, Happerfield L, Pinder SE, Balan KK, Wishart GC. P-glycoprotein expression is associated 
with sestamibi washout in primary hyperparathyroidism. Br J Surg 2007; 94: 1491-5.

11. Erbil Y, Kapran Y, Işsever H, Barbaros U, Adalet I, Dizdaroğlu F, et al. The positive effect of adenoma weight and oxyphil cell content on preoperative localization with 99mTc-sestamibi scanning for primary hyperparathyroidism. Am J Surg 2008; 195: 34-9.

12. Wu S, Hwang SS, Haigh PI. Influence of a negative sestamibi scan on the decision for parathyroid operation by the endocrinologist and surgeon. Surgery 2017; 161: 35-43.

13. Dy BM, Richards ML, Vázquez BJ, Thompson GB, Farley DR, Grant CS. Primary Hyperparathyroidism and Negative Tc99 Sestamibi Imaging: To Operate or Not?. Ann Surg Oncol 2012; 19: 2272-8.

14. Keidar Z, Solomonov E, Karry R, Frenkel A, Israel O, Mekel M. Preoperative [99mTc]MIBI SPECT/CT Interpretation Criteria for Localization of Parathyroid Adenomas-Correlation with Surgical Findings. Mol Imaging Biol 2017; 19: 265-70.

15. Palestro CJ, Tomas MB, Tronco GG. Radionuclide imaging of the parathyroid glands. Semin Nucl Med 2005; 35: 266-76.

16. Perrier N, Edeiken B, Nuñez R, et al. A novel nomenclature to classify parathyroid adenomas. World J Surg 2009; 33: 412-6.

17. Hung GU, Wang SJ, Lin WY. Tc-99m MIBI parathyroid scintigraphy and intact parathyroid hormone levels in hyperparathyroidism. Clin Nucl Med 2003; 28: 180-5.

18. Jorna F, Hollema H, Hendrikse H, Bart J, Brouwers A, Plukker J. P-gp and MRP1 expression in parathyroid tumors related to histology, weight and $(99 \mathrm{~m}) \mathrm{Tc}$-sestamibi imaging results. Exp Clin Endocrinol Diabetes 2009; 20; 117: 406-12.

19. Mehta NY, Ruda JM, Kapadia S, Boyer PJ, Hollenbeak CS, Stack BC Jr. Relationship of technetium Tc 99m sestamibi scans to histopathological features of hyper- functioning parathyroid tissue. Arch Otolaryngol Head Neck Surg 2005; 131: 493-8.

20. Nichols KJ, Tronco GG, Palestro CJ. Effect of reconstruction algorithms on the accuracy of $99 \mathrm{mTc}$ sestamibi SPECT/CT parathyroid imaging. Am J Nucl Med Mol Imaging 2015; 5: 195-203.

21. Bural G, Muthukrishnan A, Oborski M, Mountz J. Improved benefit of SPECT/CT compared to SPECT alone for the accurate localization of endocrine and neuroendocrine tumors. Mol Imaging Radionucl Ther 2012; 21: 91-6.

22. Carlier T, Oudoux A, Mirallié E, Seret A, Daumy I, Leux C, et al. 99mTc-MIBI pinhole SPECT in primary hyperparathyroidism: comparison with conventional SPECT, planar scintigraphy and ultrasonography. Eur J Nucl Med Mol Imaging 2008; 35: 637-43.

23. Patel, C.N, Salahudeen HM, Lansdown M, Scarsbrook AF. Clinical utility of ultrasound and $99 \mathrm{~m}-\mathrm{Tc}$ sestamibi SPECT/CT for preoperative localization of parathyroid adenoma in patients with primary hyperparathyroidism. Clin Radiol 2010; 65: 278-87.

24. Roy M, Mazeh H, Chen H, Sippel RS. Incidence and localization of ectopic parathyroid adenomas in previously unexplored patients. World J Surg 2013; 37: 102-6.

25. Neumann DR, Esselstyn CB, Maclntyre WJ, Go RT, Obuchowski NA, Chen EQ, et al. Comparison of FDGPET and sestamibi-SPECT in primary hyperparathyroidism. Nucl Med 1996; 37: 1809-15.

26. Kluijfhout WP, Pasternak JD, Drake FT, Beninato T, Gosnell J, Shen W, et al. Use of PET tracers for parathyroid localization: a systematic review and meta-analysis. Langenbeck's Archives of Surgery 2016; 401: 925-35.

27. Paul A, Villepelet A, Lefèvre M, Périé S. Oncocytic parathyroid adenoma. Eur Ann Otorhinolaryngol Head Neck Dis 2015; 132: 301-3. 